\title{
Determination of effective photon lifetime in nitrogenic laser in one and two dimension
}

\author{
S. N. Hosseinimotlagh ${ }^{1}$, M. T. Yazdani ${ }^{1}$, H. Zare ${ }^{2}$ \\ ${ }^{1}$ Department of Physics, Shiraz branch Islamic Azad University, Shiraz, Iran \\ ${ }^{2}$ Department of Physics, Science and Research Branch, Islamic Azad University, Fars, Iran
}

\section{Email address:}

hoseinimotlagh@hotmail.com (S. N. Hosseinimotlagh)

\section{To cite this article:}

S. N. Hosseinimotlagh, M. T. Yazdani, H. Zare. Determination of Effective Photon Lifetime in Nitrogenic Laser in One and Two Dimension. Optics. Vol. 3, No. 1, 2014, pp. 5-11. doi: 10.11648/j.optics.20140301.12

\begin{abstract}
Since one of the most valuable measurable parameters in laser, called effective cavity lifetime, gives useful information about laser, this paper aims to study the description of it dependency, $\tau_{p h}^{e f f}$, on geometrical characteristics of $\mathrm{N}_{2}$-laser, electrodes length and amplifier gap separation. First based on the studies carried out on it, an oscillator-amplifier laser is used which operates under moderate current density conditions; Then in order to obtain a theoretical relation for effective cavity lifetime and to demonstrate the mentioned dependency using rate equations, at first a one-dimensional method is used for the photon density. Since the answers of rate equations in an oscillator-amplifier laser are complicated, a single-oscillator based modelis offered to make rate equations simpler. In this model, at first it is supposed that the photon density of inner part of the amplifier could ben $\mathrm{n}_{\mathrm{ph}}(\mathrm{z}, \mathrm{t})=\mathrm{n}_{\mathrm{ph}}(0, \mathrm{t}) \exp \left(\mathrm{g}_{0}(\mathrm{z}) \mathrm{z}\right)$, If $\mathrm{n}_{\mathrm{ph}} \cong \mathrm{n}_{\mathrm{ph}}(\mathrm{z}, \mathrm{t})$, then rate equations are used for this density and since $\mathrm{g}_{0}$ is a function of $\mathrm{z}$ or amplifier electrode length $\left(\mathrm{Z} \cong \mathrm{l}_{\mathrm{AMP}}\right)$, the cavity effective life time is calculated for equivalent oscillator.Then ,Since most of studies carried out in one dimension, so for approaching to more actual system a two -dimensional method is used for the photon density. So, we consider $Z$ andY, which $Z$ is along amplifier electrodes length and $\mathrm{Y}$ is along gaps separation. Supposing that $\mathrm{Z}$ and $\mathrm{Y}$ are independent on the photon density, two independent relations can be considered for the photon density. In this step, 2-dimensional photon density could be regarded as: $\mathrm{n}_{\mathrm{ph}}(\mathrm{z}, \mathrm{y}, \mathrm{t})=\mathrm{n}_{\mathrm{ph}}(\mathrm{z}, \mathrm{t}) \mathrm{n}_{\mathrm{ph}}(\mathrm{y}, \mathrm{t})$. and then 2-dimensional effective cavity lifetime amount is obtained as: $\left(\tau_{\text {eff }}^{p h}\right)^{-1}=\frac{c}{l_{A M p}}\left(1+Y_{l}^{z}+b l_{A M p} Y_{l}^{Z}\right)+\frac{c}{d_{A M p}}\left(Y_{l}^{y}+a d_{A M p} Y_{l}^{y}\right)$, This relation includes 2 independent values along the electrodes length $\left(\mathrm{Z} \cong \mathrm{l}_{\mathrm{AMP}}\right)$ and gap separation $\left(\mathrm{y} \cong \mathrm{d}_{\mathrm{AMP}}\right)$. It also demonstrates that the obtained 2-dimentional relation represents a perfect schema for lifetime behavior. The results of this calculation are consistent with other reported N2-laser effective cavity lifetime values measured under moderate current density conditions.
\end{abstract}

Keywords: Laser, Photon, Nitrogenic, Lifetime, Effective

\section{Introduction}

\subsection{Historical Background}

The milestones in the history of lasers dates back 100 years when Einstein came up with the idea of stimulated emissions, which became a platform for the Nobel prize winner T. H. Maiman in inventing the first ever known laser in 1960 . The first gas laser was developed by A. Javan, W. Benneth and D. Harriott of Bell laboratories in 1961 using a mixture of helium and neon gases before Heard demonstratedthe action of the first nitrogen laser in 1963. Although the average power of this laser was very low, it captured people's attention to develop new design methodologies to improve the peak power output. Research continued until a transverse electrical discharge at atmospheric pressure (TEA) nitrogen laserwas developed which was capable of producing megawatt powers using molecular nitrogen at atmospheric conditions. This appeared at a time when it was needed in ultraviolet laser development, which led to excimer lasers which are now of great use on large scale [15]. Since then, lasers have developed enormously with a wide range of applications in industries, military, medical, and academic research. Today, laser devices have numerous applications in communication systems, in supermarkets as check \{out scanners, in photocopying and scanning machines, in home compact disc players and CDs, and many others. There are various types of lasers that have been developed so far with a wide range of physical and operating parameters, 
characterized by their state of lasing material such as solidstate, liquid or gas.

\subsection{Motivation}

Nitrogen molecules are the most abundant molecules in the earth's atmosphere, constituting 78:08\%.Electron collisions with molecular nitrogen continue to receive interest due to their importance in gaseous discharge processes, such as those involved in nitrogen lasers. Nitrogen lasers are capable of producing very high power short pulses of ultraviolet light at a wavelength of $337.1 \mathrm{~nm}$. Therefore their studies play an important role in laser technology. They have a wide range of applications in dye laser pumping,lidar (remote sensing), fast speed photography, atomic and lifetime spectroscopy, medical and biological research, etc. Compared to other gas lasers, nitrogen lasers are very cheap and easy to construct. Thisexplains why they are sometimes called home built lasers". However, their applications are limited by very poor efficiency, which does not often exceed $1 \%$. A surprising report of higher efficiencies of about 3\% was reported by Oliveira et al. [11]. As long as nitrogen lasers have been in the field, theoretical and experimental investigations on various parameters and electronic discharge circuit designs continue in order to improve their efficiencies. Thus, following the previous work done in [13, $11,10]$, it has been shown that nitrogen lasers operate efficiently, depending on a fast discharge circuit to provide a high voltage across the laser tube, anterior to the gas breakdown.

\section{Pumping Mechanism for a Nitrogen Laser}

A nitrogen molecule like any other diatomic molecule possesses vibronic energy levels that constitute both vibrational and electronic states. These energy states are mainly separated by the change in electron energy of the atoms, and a small contribution resulting from the vibrations within the nitrogen molecules themselves. The laser action therefore involves a series of the transitions, resulting from the changes in vibrational and electronic states of the nitrogen molecules, which are nearly spaced to favor the emission of ultraviolet radiation at $337.1 \mathrm{~nm}$ [2]. The pumping mechanism in a nitrogen laser, involves collision of high energy electrons with the gas molecules in a laser tube through electron impactexcitation. The accelerated electrons in the laser tube strike the nitrogen molecules, thus exciting them to a higher electronic energy state. Figure 1.5shows the energy level scheme for a nitrogen laser, with each level showing a series of vibrational energy level, which depends on the internuclear separation of the molecule. The laser normally starts when the nitrogen molecules become excited by an electric discharge in the lasing tube from the electronic ground state, labeled $X^{1} \Sigma_{g}^{+}$energy band to the upper lasing level, labeled $C^{3} \Pi_{v}$ energy band. The molecules at the upper lasing level are unstable, thus they decay to the lower lasing level, labeled as $B^{3} \Pi_{g}$ energy band, by emitting photons of ultraviolet radiation at $337.1 \mathrm{~nm}$. This corresponds to only energy levels with the lowest vibration state, for which $v=0$, and $v=1$ corresponding to the upper vibrational state. However, other transition states are also possible. For example in a 1-0 state, where in this case the lower lasing level involved in the vibration state is at $v=1$ and the upper lasing level is the same as the lowest, at $v=0$ [2]. This result into a shorterjump during the transition state than before, thus an output with a lower energy at a wavelength of $358 \mathrm{~nm}$ is given off as shown in Figure 1.5. When a molecule emits a photon as it falls to the lower lasing level, it then decays to a metastable state where it stays. Thus a nitrogen laser is effectively a three-level laser.

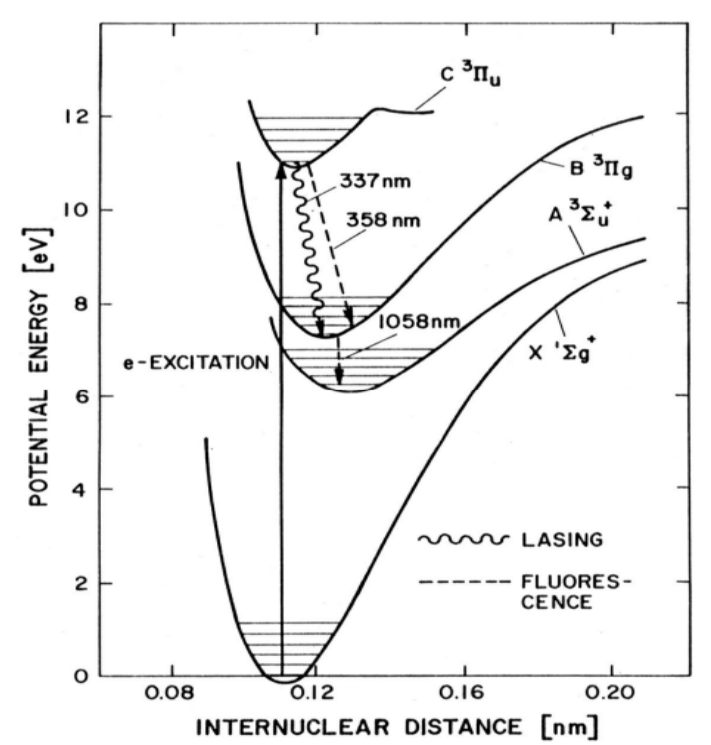

Figure 1.5. Energies of the molecular nitrogen, as a function of inter nuclear separation relevant for the nitrogen laser [15].

The biggest problem with a nitrogen laser is the lifetime of the upper lasing level, which is their greatest barrier in the laser development. The transition lifetime of the gas molecules from the upper lasing level to the lower lasing level is very short ( $\leq 40 \mathrm{~ns}$ ) as compared to lifetime for the transition between the lower lasing level and the meta stable state (about $10 \mu s$ ) [2]. Thus the population of the gasmolecules at the lower level exceeds that at the upper lasing level. This means that the lasing process will terminate, since the condition for lasing to take place is violated. Creating a population inversion at the upper lasing level in this case, is only possible if the pump power from the source is sufficient to have the nitrogen molecules in the upper lasing level as quick as possible. Gas lasers in general, arepumped directly by electron-impact excitation. The gas molecules are excited from the ground level to the upper level through collisions with the electrons accelerated by the electric field set-up between the electrodes of the discharge chamber.Two types of gas discharge are commonly used for the N2-laser excitation. They are transversal and longitudinal discharges. High laser output powers can usually be obtained by the use of 
transversal discharge of the types transversely excited (TE), and transversely excited atmospheric pressure (TEA) nitrogen lasers. The alternative approach is the longitudinal discharge scheme (LE) which isappropriate to be used at the low gas pressure operational regime. The advantage of longitudinaldischarge devices is the discharge uniformity that can be easily achieved at gas pressures of a few Torr. In addition, a low beam divergence and good circular mode structure of Gaussian type can be expected from the LE configurations. The output energy of lasers based on the longitudinally excited mechanism, however, is limited in the order of tens of $1 \mathrm{~J}$ and that is mainly due to the higher impedanceof the gas discharge compared to that of transversal discharges. On the other hand, for improving the laser beam quality, enhancement of the laser output energy, and reducing the laser beam divergence, designs based on oscillator-amplifier(OSC-AMP) N2-lasers have also been proposed. These include TEA-TEA, TE-TE, and the combined TEA-TE oscillator-amplifier systems [1-12] In a laser system consists of an OSC-AMP, in addition to achieving improvement in laserperformances, it gives also a method of probing the laser output extracted from amplifier as the input energy to the amplifier changes. This technique is commonly used for measurements of the small signal gain, g0, and saturation energy density,Es, of the amplifier section.

\section{Theoretical Considerations}

Based on studies that have been doing on the N-2 lasers characteristics, we introduce a method to obtain an analytical expression for the effective cavity lifetime. For our study purposes we consider an oscillator amplifier (Ose-Amp) laser system, Details of theoretical approach including the electric-circuit behavior and the rate equations for an OSC-AMP laser system usinga Blumlein circuit have been reported previously [14]. Briefly In fact, in the theoretical approach it was shown that in an OSCAMP laser system, where the solution to the relevant equations (i.e. the equivalent electric circuit, and the rate equations) become complicated, a model based on introducing an equivalent N2- laser system to apply as a single traveling wave oscillator can be used to simplify the equations and consequently to reach the appropriate results, compatible with the measurements. For applying the model it was required to use an open-circuit OSC condition in the real OSC-AMP laser system both through the experiment and the data reduction procedure, for obtaining the electriccircuit parameters. Thiswas simply obtained by letting $I_{3}=$ 0 , where $I_{3}$ is the current corresponding to theOSC section of the OSC-AMP electric-circuit system. The advantages of using the equivalent OSC-laser system instead of applying a complicated real OSC-AMP configuration are as follows:

1. We can apply the rate equations and the equivalent electric-circuit for a single OSC, where the relevant equations for an OSC laser system have been realized in the past [4], and almost in recent years [12]; and also with the proposed model, the approach for the numerical solution to the equations is easily attainable.

2. For evaluating the equivalent electric-circuit parameters, such as resistance and inductance of the laser system, it is required to observe the voltage waveforms corresponding to the relevant laser charging capacitors. For this purpose it is only needed to use a open-circuit condition of $I_{3}=0$ for the OSC section in the OSC-AMP laser system, and the analysis of the waveforms corresponding to charging capacitors of $\mathrm{C} 1$ and $\mathrm{C} 2$ can be performed without any complications using the relevant equations given in Refs. [14,12].

3. It has shown that it is not necessary to drop some terms such as spontaneous emission and pumping rate in the rate equations which is commonly appeared in the literature [5]. In fact simulation studies have proved that keeping these terms in the rate equations will affect the calculated results and consequently it was realized that they cannot be simply ignored.

In the present study we are extending the studies that have been done on understanding the effective cavity lifetime behavior in the N2-laser. For completing the one dimensional traveling wave model with more accurate results Section 3.1has been introduced. To finalize our work in this respect a model based on a2-dimensional approach for the photon density is given in Section 3.2. In this approach the electrodes gap separation of the amplifier, $d_{A M P}$, will appear in the effective cavity lifetime formulation. Thus, the dependency of the N2-laser lifetime on $l_{A M P}$ and $d_{A M P}$, where they are referring to the laser geometricalconfigurations will be introduced in this section

\subsection{One Dimensional Model}

Following the rate equations given by Fitzsimmons et al. [3], therequired equations for the $C^{3} \prod_{u}$ and $B^{3} \prod_{g}$ states along with theequation for the photon density are given by

$$
\begin{gathered}
\frac{d N_{c}}{d t}=n_{e} N_{g a s} \int_{0}^{\infty} g\left(T_{e}, v\right) \sigma_{c}(v) 4 \pi v^{3} d v-\sigma_{s t i m} n_{p h} c\left(N_{C}-N_{B}\right)-\frac{N_{C}}{\tau_{C}} \\
\frac{d N_{B}}{d t}=n_{e} N_{g a s} \int_{0}^{\infty} g\left(T_{e}, v\right) \sigma_{B}(v) 4 \pi v^{3} d v+\sigma_{s t i m} n_{p h} c\left(N_{C}-N_{B}\right)-\frac{N_{B}}{\tau_{B}}+\frac{N_{C}}{\tau_{C}} \\
\frac{\mathrm{dn} \mathrm{ph}}{\mathrm{dt}}=\sigma_{\text {stim }} \mathrm{n}_{\mathrm{ph}} \mathrm{C}\left(\mathrm{N}_{\mathrm{C}}-\mathrm{N}_{\mathrm{B}}\right)-\frac{\mathrm{n}_{\mathrm{ph}}}{\tau_{\mathrm{ph}}}+\delta \frac{\mathrm{N}_{\mathrm{C}}}{\tau_{\mathrm{C}}}
\end{gathered}
$$

For the small signal gain and the output energy extracted fromthe gain medium, we have

$$
g_{0}=\sigma_{\text {stim }}\left(\mathrm{N}_{\mathrm{C}}-\mathrm{N}_{\mathrm{B}}\right)
$$

$$
\varepsilon_{\text {out }}=n_{\text {ph }} h v V_{\text {dis }}
$$

where in Eqs. (1) and (2), $N_{\text {gas }}$ is the ground-state gas density; $\sigma_{c}$ and $\sigma_{B}$ are the electron impact cross-sections of 
the $\mathrm{N} 2(\mathrm{C})$ and $\mathrm{N} 2(\mathrm{~B})$ states, respectively; $g\left(T_{e}, v\right)$ is the normalized Maxwell-Boltzmanndistribution; $\sigma_{\text {stim }}$ is the stimulated emission cross-section and is given by

$\lambda^{2} / 8 \pi \Delta v \tau_{c} \approx 4.5 \times 10^{-15} \mathrm{~cm}^{2}$ for $\Delta v=278 \mathrm{GHZ} \quad$ [6] $\tau_{C}$ and $\tau_{B}$ (40 ns and $6 \mu S$ respectively) are the spontaneous lifetime of thegeometrical factor for spontaneous emission; and $V_{d i s}=S l_{A M P} d_{A M P}$ is the discharge volume. Referring to the theory of pulse propagation. In a laser amplifier, the left-hand side of Eq. (1c) has to modifiedto a right-traveling propagation pulse of the form $\frac{\partial n_{p h}}{\partial t}+C \frac{\partial n_{p h}}{\partial z}$, where the zdependence of the photon density has beenconsidered [1], and also by substituting $\mathrm{Eq}(2 \mathrm{a})$ in $\mathrm{Eq}(1 \mathrm{c})$, For a rough estimation of $\tau_{p h}^{e f f}$ if we ignore the effectof spontaneous emission which appears as the third term inEq. (1c), we have

$$
\frac{\partial \mathrm{n}_{\mathrm{ph}}}{\partial \mathrm{t}}+C \frac{\partial n_{p h}}{\partial z}=C g_{0} n_{p h}-\frac{n_{p h}}{\tau_{p h}}
$$

Where $n_{p h} \cong n_{p h}(z, t)$ is the photon density corresponding to photonstraveling in the z-direction [9], $\tau_{p h}$ is the photon lifetime in the amplifier.By introducing the equivalent OSC system, we have furtherassumedthat photon density inside the AMP section can be written as

$$
n_{p h}(z, t)=n_{p h}(0, t) e^{g_{0}(z) z}
$$

In the theory of pulse amplification the above equation refers to the photon density growth rate which is given by the exponential law in the limit of infinitesimal pulse width [5]. By substituting Eq. (4) in Eq. (3), and by considering that $g_{0}$ is also a function of $\mathrm{z}$, or the length of the amplifying medium $\left(z \cong l_{A M P}\right)$, Eq (3) changes to

$$
\frac{\partial \mathrm{n}_{\mathrm{ph}}(\mathrm{z}, \mathrm{t})}{\partial \mathrm{t}}+\mathrm{C}\left(\frac{1}{\mathrm{~L}_{\mathrm{AMP}}}+\left|\frac{\partial \mathrm{g}_{0}}{\partial \mathrm{L}_{\mathrm{AMP}}} \mathrm{L}_{\mathrm{AMP}}\right|\right) \mathrm{n}_{\mathrm{ph}}(\mathrm{z}, \mathrm{t})=0
$$

According this relation the cavity effective lifetime for the introduced OSC will be obtained

$$
\frac{1}{\tau_{\mathrm{ph}}^{\mathrm{eff}}}=C\left\{\frac{1}{\mathrm{~L}_{\mathrm{AMP}}}+\left|\frac{\partial \mathrm{g}_{0}}{\partial \mathrm{L}_{\mathrm{AMP}}} \mathrm{L}_{\mathrm{AMP}}\right|\right\}
$$

Also, a further remark on the mean cavity lifetime $\tau_{p h}$ should be given at this stage. For a traveling wave excitation, we can write, $\tau_{p h}=\frac{l_{A M P}}{C}$ this relation has already been introduce by ChangandTeii[7,14]. For simplifying an OSC-AMP calculation, and byapplying a model base on a single OSC, without considering anymirrors reflectivity and cavity losses, $\tau_{p h}$ has to be modified. Theapproach for this modification relies on experimental observations,we can realizethat for short electrode lengths $\tau_{p h}$ needsto be somehow smaller than $\frac{l_{A M P}}{C}$ to get a betteragreement withthe experimental observations. observations are indicatingthat for this purpose we can introduce the effective cavity lifetime $\tau_{p h}^{\text {eff }}=k \frac{l_{A M P}}{C}$. Furthermore, in the equivalent OSC system at the lasing threshold we have related $g_{0} l_{A M P}$ to the power loss, given by $\gamma_{l}=\gamma_{l}^{\max } e^{-b l_{A M P}}$, where $\gamma_{l}^{\max }$ is themaximum power loss and $\mathrm{b}$ is a constant and with some approximationit has been determined by the simulation and experiment [14].So with these considerations we can get a new relation for the effective cavity lifetime. Note that because of the term appeared inside the absolute value we have two values for $\tau_{p h}^{\text {eff }}$,

$$
\begin{gathered}
\tau_{p h}^{\text {eff }}=\frac{\mathrm{l}_{\mathrm{AMP}}}{\mathrm{C}\left(1+\gamma_{1}-\mathrm{l}_{\mathrm{AMP}} \frac{\partial \gamma_{1}}{\partial \mathrm{l}_{\mathrm{AMP}}}\right)}=\frac{\mathrm{l}_{\mathrm{AMP}}}{\mathrm{C}\left(1+\gamma_{1}+\mathrm{b} \gamma_{1} \mathrm{l}_{\mathrm{AMP}}\right)} \\
\tau_{p h}^{\text {eff }}=\frac{\mathrm{l}_{\mathrm{AMP}}}{\mathrm{C}\left(1-\gamma_{1}+\mathrm{l}_{\mathrm{AMP}} \frac{\partial \gamma_{1}}{\partial \mathrm{l}_{\mathrm{AMP}}}\right)}=\frac{\mathrm{l}_{\mathrm{AMP}}}{\mathrm{C}\left(1-\gamma_{1}-\mathrm{b} \gamma_{1} \mathrm{l}_{\mathrm{AMP}}\right)}
\end{gathered}
$$
by

As you can see there are two values for $\mathrm{k}$ that are given

$$
\begin{aligned}
& k^{(1)}=\frac{1}{1+\gamma_{1}+\mathrm{b} \gamma_{1} \mathrm{l}_{\mathrm{AMP}}} \\
& k^{(2)}=\frac{1}{1-\gamma_{1}-\mathrm{b} \gamma_{1} \mathrm{l}_{\mathrm{AMP}}}
\end{aligned}
$$

But it seems that justEqs(7a) and also (8a) are in good agreement withmeasurement and results that are available based on studies that had been done on N-2laser parametersobservations are indicating [8] that $\mathrm{k}$ is a positive number and is less than 1 . In fig .1 .we have shown the behavior of $k^{(1)}$ and $k^{(2)}$ vs. $l_{\mathrm{AMP}}$, here $\gamma_{1}^{\max }=4.193$ and $b=0.042\left(\mathrm{~cm}^{-1}\right)$ have been used.

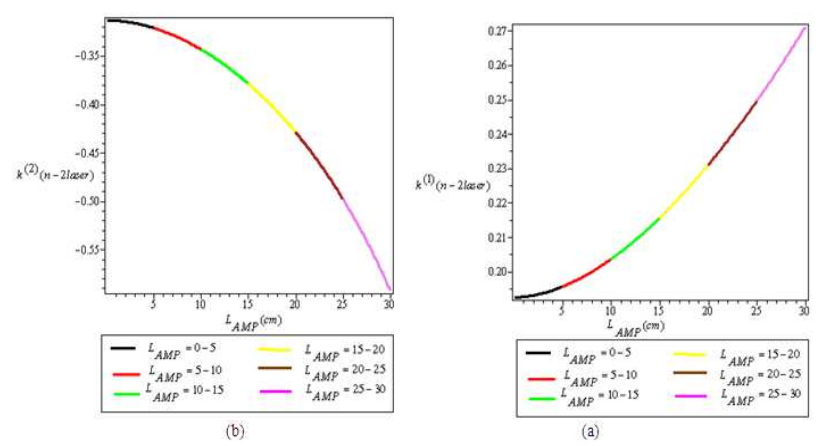

Fig 1. Plots corresponding to single dimensional model : (a) $k^{(1)}$ as given by $E q(8 a)$ and (b) $k^{(2)}$ as given by (8b) vs. $l_{A M P}$.In both figures the parameters given to the corresponding equations deduced from [ 8$]$.

Plot (a) shows $k^{(1)}$ increases as the electrode length of amplifier increases, it's a positive value that dose not exceed from one. plot (b) shows $k^{(2)}$ reduces as $\mathrm{l}_{\mathrm{AMP}}$ increases and it's a negative value, so it will be ignored simplyfor amplifiers of long electrode lengths, which are usually used in any laser laboratory, we can apply $\tau_{p h}^{\text {eff }}=\frac{l_{A M P}}{C}$,i.e., $k=1$. For amplifiers of short electrode lengths, however, the effective cavity lifetime of $\tau_{p h}^{e f f}=k \frac{l_{A M P}}{C}$ appropriate $\mathrm{k}$ parameter has to applied. The profile corresponding to $\tau_{p h}^{\text {eff }}$ is given in Fig.2. parameters $\gamma_{1}^{\max }=4.193, b=0.042\left(\mathrm{~cm}^{-1}\right)$ labeled by (Data1) and $\gamma_{1}^{\text {z,max }}=3.89 \quad, b=0.06 \mathrm{~cm}^{-1}$ labeled by (Data2) that have been used for drawing $\tau_{p h}^{\text {eff }}$ profile are based on experimental measurements. 


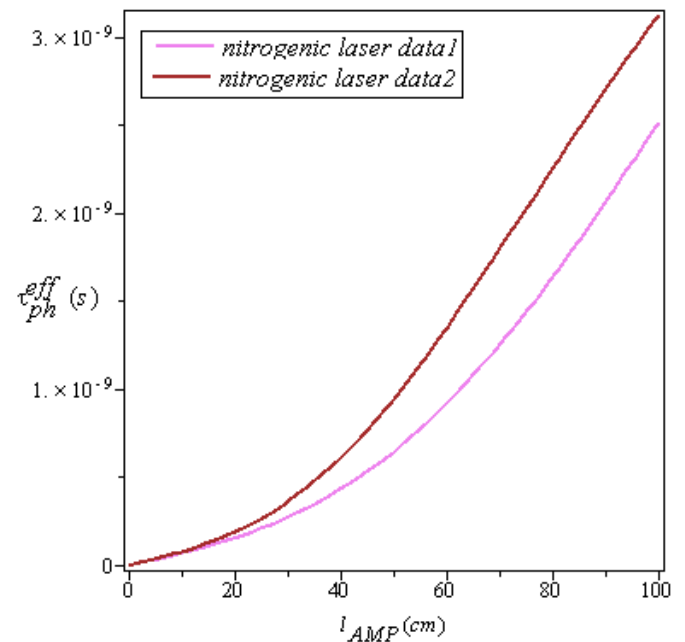

Fig2. Plot of $\tau_{p h}^{\text {eff }}$ vs. $l_{\text {AMP }}$. Data 1 deduced from [8] and Data 2 deduced from [7].

Plots are indicating that, $\tau_{p h}^{e f f}$ is increasing as $l_{A M P}$ increases.

\subsection{Two Dimensional Model}

For describing this model two directions; $\mathrm{z}$ and $\mathrm{y}$,chosen parallel to the optical axisand electrodes direction, respectively, are used. while the whole system as atraveling wave OSC must be faced with a single value for the photon lifetime given by $\tau_{p h}=\frac{l_{A M P}}{C}$, where it corresponds to the zdirection, i.e., to the direction of the laser output beam. Thus, with the assumption of $\mathrm{z}$ and $\mathrm{y}$ independency of the photon density, we can rewrite two independent equations for the photon density, i.e.

$$
\begin{gathered}
\frac{\partial \mathrm{n}_{\mathrm{ph}, \mathrm{z}}(\mathrm{z}, \mathrm{t})}{\partial \mathrm{t}}+C \frac{\partial \mathrm{n}_{\mathrm{ph}, \mathrm{z}}(\mathrm{z}, \mathrm{t})}{\partial \mathrm{z}}=\mathrm{C} g_{0 z} \mathrm{n}_{\mathrm{ph}, \mathrm{z}}(\mathrm{z}, \mathrm{t})-\left(\frac{\mathrm{n}_{\mathrm{ph}, \mathrm{z}}(\mathrm{z}, \mathrm{t})}{\tau_{\mathrm{ph}}}\right) \\
\frac{\partial \mathrm{n}_{\mathrm{ph}, \mathrm{y},(\mathrm{y}, \mathrm{t})} \partial \mathrm{t}}{\partial \mathrm{t}} \frac{\partial \mathrm{n}_{\mathrm{ph}, \mathrm{y}}(\mathrm{y}, \mathrm{t})}{\partial \mathrm{y}}=\mathrm{C} g_{0 y} \mathrm{n}_{\mathrm{ph}, \mathrm{y}}(\mathrm{y}, \mathrm{t})
\end{gathered}
$$

where in Eq. (9a), in contrast to Eq. (9b), we dropped the term corresponding to the photon-lifetime in the y-direction, otherwise its presence leads to an inconsistency with the measurements. Thus, up to this stage two independent gainvalues corresponding to $\mathrm{z}$ and $\mathrm{y}$-directions, i.e. $\mathrm{g} 0 \mathrm{z}$ and $\mathrm{g} 0 \mathrm{y}$ are introduced, as given by Eqs (9a) and (9b).

Multiplying Eq. (9a) by $\mathrm{n}_{\mathrm{ph}, \mathrm{y}}(\mathrm{y}, \mathrm{t})$, and Eq. (9b) by $\mathrm{n}_{\mathrm{ph}, \mathrm{z}}(\mathrm{z}, \mathrm{t})$, we have

$$
\begin{aligned}
& \frac{\partial \mathrm{n}_{\mathrm{ph}, \mathrm{z}}(\mathrm{z}, \mathrm{t})}{\partial \mathrm{t}} \mathrm{n}_{\mathrm{ph}, \mathrm{y}}(\mathrm{y}, \mathrm{t})+C \frac{\partial \mathrm{n}_{\mathrm{ph}, \mathrm{z}}(\mathrm{z}, \mathrm{t})}{\partial \mathrm{z}} \mathrm{n}_{\mathrm{ph}, \mathrm{y}}(\mathrm{y}, \mathrm{t})=\mathrm{C} g_{0 \mathrm{z}} \mathrm{n}_{\mathrm{ph}, \mathrm{z}}(\mathrm{z}, \mathrm{t}) \mathrm{n}_{\mathrm{ph}, \mathrm{y}}(\mathrm{y}, \mathrm{t})-\left(\frac{\mathrm{n}_{\mathrm{ph}, \mathrm{y}}(\mathrm{y}, \mathrm{t}) \mathrm{n}_{\mathrm{ph}, \mathrm{z}}(\mathrm{z}, \mathrm{t})}{\tau_{\mathrm{ph}}}\right) \\
& \frac{\partial \mathrm{n}_{\mathrm{ph}, \mathrm{y}, \mathrm{t}, \mathrm{t})}}{\partial \mathrm{t}} \mathrm{n}_{\mathrm{ph}, \mathrm{z}}(\mathrm{z}, \mathrm{t})+\mathrm{C} \frac{\partial \mathrm{n}_{\mathrm{ph}, \mathrm{y}}(\mathrm{y}, \mathrm{t})}{\partial \mathrm{y}} \mathrm{n}_{\mathrm{ph}, \mathrm{z}}(\mathrm{z}, \mathrm{t})=\mathrm{C} g_{0 y} \mathrm{n}_{\mathrm{ph}, \mathrm{y}}(\mathrm{y}, \mathrm{t}) \mathrm{n}_{\mathrm{ph}, \mathrm{z}}(\mathrm{z}, \mathrm{t})
\end{aligned}
$$

Then by adding two produced equations and with assumption of: $n_{p h}(z, y, t)=n_{p h, z}(z, t) n_{p h, y}(y, t) a$ new relation will acquire

$$
\frac{\partial \mathrm{n}_{\mathrm{ph}}(\mathrm{z}, \mathrm{y}, \mathrm{t})}{\partial \mathrm{t}}+C \frac{\partial \mathrm{n}_{\mathrm{ph}}(\mathrm{z}, \mathrm{y}, \mathrm{t})}{\partial \mathrm{z}}+C \frac{\partial \mathrm{n}_{\mathrm{ph}}(\mathrm{z}, \mathrm{y}, \mathrm{t})}{\partial \mathrm{y}}=C\left(g_{0 z}+g_{0 y}\right) \mathrm{n}_{\mathrm{ph}}(\mathrm{z}, \mathrm{y}, \mathrm{t})-\frac{\mathrm{n}_{\mathrm{ph}}(\mathrm{z}, \mathrm{y}, \mathrm{t})}{\tau_{\mathrm{ph}}}
$$

with the following assumptions:

$$
\nabla_{t}=\frac{\partial}{\partial z}+\frac{\partial}{\partial y} \text { and } \mathrm{g}_{0}=\mathrm{g}_{0 \mathrm{z}}+\mathrm{g}_{0 \mathrm{y}}
$$

we can write

$$
\frac{\partial \mathrm{n}_{\mathrm{ph}}(\mathrm{z}, \mathrm{y}, \mathrm{t})}{\partial \mathrm{t}}+C \nabla_{t} \mathrm{n}_{\mathrm{ph}}(\mathrm{z}, \mathrm{y}, \mathrm{t})=C g_{0} \mathrm{n}_{\mathrm{ph}}(\mathrm{z}, \mathrm{y}, \mathrm{t})-\frac{\mathrm{n}_{\mathrm{ph}}(\mathrm{z}, \mathrm{y}, \mathrm{t})}{\tau_{\mathrm{ph}}}
$$

For evaluating the cavity effective lifetime, we can repeat our argument as in the previous section, or equivalently by introducing the overall photon density in an equivalent two-dimensional OSC; so

$$
\mathrm{n}_{\mathrm{ph}}(\mathrm{z}, \mathrm{y}, \mathrm{t})=\mathrm{n}_{\mathrm{ph}}(0,0,0) e^{\frac{\mathrm{-t}}{\tau_{\mathrm{ph}}^{\text {eff }}}} \exp \left[g_{0 z}\left(\mathrm{z}, \mathrm{r}_{1}^{\mathrm{z}}\right) \mathrm{z}+g_{0 y}\left(y, r_{1}^{\mathrm{y}}\right) y\right]
$$

Where, we assumed that $g_{0 z}$ and $g_{0 y}$ are functions ofz, $Y_{1}^{\mathrm{z}}$; and $y, Y_{1}^{\mathrm{y}}$, respectively. $Y_{1}^{\mathrm{z}}$ and $\gamma_{1}^{\mathrm{y}}$ are two independent power losses correspond to $\mathrm{z}$ and $\mathrm{y}$-directions, respectively. For the power loss,$Y_{l}^{z}$, in the $\mathrm{Z}$-direction we can write

$$
Y_{1}^{z}=Y_{1}^{z, \max } e^{-b z} g\left(z=l_{\text {AMp }}\right)
$$

Where $\gamma_{1}^{\text {z,max }}$ and $b$ are constants that can be determined experimentally.For the $y$-direction, we can also write analogous to the z-direction :

$$
Y_{1}^{\mathrm{y}}=Y_{1}^{y, \text { max }} \mathrm{e}^{-\mathrm{ay}} g\left(y=d_{\mathrm{AMP}}\right)
$$

Where again $\gamma_{1}^{y \text {,max }}$ and a are introduced as constants. The behavior of $Y_{1}^{y}$ as given by Eq. (15)was found that it is consistent with the experiment. Plots of $Y_{1}^{z}$ and $Y_{1}^{\mathrm{y}}$ Corresponding to Eq.(13) and (14) are given in Fig.3.
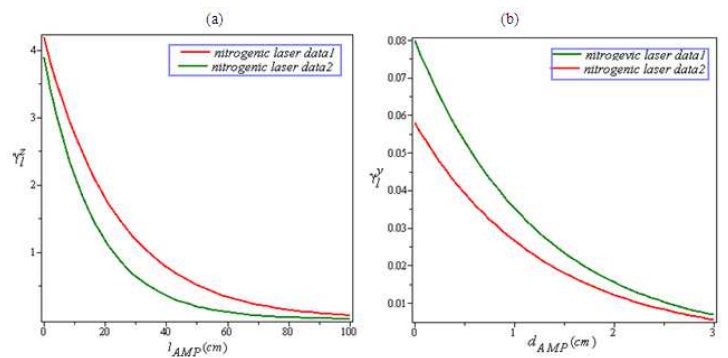

Fig3. (a) plot of $Y_{l}^{z}$ vs. $l_{A M p}$ and (b) plot of $Y_{l}^{y}$ vs. $d_{A M P}$. 
Upon substituting Eq. (13) into Eq. (11), we have

$$
\begin{gathered}
\frac{\partial \mathrm{n}_{\mathrm{ph}}(\mathrm{z}, \mathrm{y}, \mathrm{t})}{\partial \mathrm{t}}+\left[\frac{\mathrm{C}}{\mathrm{z}}+\mathrm{Cz}\left|\frac{\partial \mathrm{g}_{0 \mathrm{z}}}{\partial \mathrm{z}}\right|+\mathrm{Cy}\left|\frac{\partial \mathrm{g}_{0 \mathrm{y}}}{\partial \mathrm{y}}\right|\right] \mathrm{n}_{\mathrm{ph}}(\mathrm{z}, \mathrm{y}, \mathrm{t})=0 \\
\frac{1}{\tau_{\mathrm{ph}}^{\mathrm{eff}}}=\frac{\mathrm{C}}{\mathrm{z}}+\mathrm{Cz}\left|\frac{\partial \mathrm{g}_{0 \mathrm{z}}}{\partial \mathrm{z}}\right|+\mathrm{Cy}\left|\frac{\partial \mathrm{g}_{0 \mathrm{y}}}{\partial \mathrm{y}}\right|
\end{gathered}
$$

At the threshold, we can introduce the following two equations,corresponding to two differentdirections

$$
\begin{aligned}
& \mathrm{g}_{0 \mathrm{z}}^{\mathrm{th}} \mathrm{z}=\gamma_{\mathrm{l}}^{\mathrm{z}}, \gamma_{\mathrm{l}}^{\mathrm{z}}>0 \\
& \mathrm{~g}_{0 \mathrm{y}}^{\mathrm{th}} y=\gamma_{l}^{y}, \gamma_{l}^{y}>0
\end{aligned}
$$

And by use ofEqs. (18a) and (18b), we can evaluate the last two terms appeared in Eq.(17),i.e.

$$
\begin{aligned}
& C z\left|\frac{\partial g_{0 z}^{t h}}{\partial z}\right|=C z\left|\frac{\mathrm{z} \partial \gamma_{1}^{\mathrm{z}} / \partial \mathrm{z}-\gamma_{1}^{\mathrm{z}}}{\mathrm{z}^{2}}\right|=\mathrm{C}\left(\frac{\gamma_{1}^{\mathrm{z}}+\mathrm{bz} \gamma_{1}^{\mathrm{z}}}{\mathrm{z}}\right), \mathrm{z}=\mathrm{l}_{\mathrm{AMP}} \\
& \mathrm{Cy}\left|\frac{\partial g_{0 y}^{t h}}{\partial y}\right|=\mathrm{Cy}\left|\frac{\mathrm{y} \partial \gamma_{1}^{\mathrm{y}} / \partial \mathrm{z}-\gamma_{1}^{\mathrm{y}}}{\mathrm{y}^{2}}\right|=\mathrm{C}\left(\frac{Y_{1}^{\mathrm{y}}+a y Y_{1}^{\mathrm{y}}}{y}\right), \mathrm{y}=\mathrm{d}_{\mathrm{AMP}}(
\end{aligned}
$$
have

Thus by substituting Eqs.(19) and (19b) in Eq.(17) we

$$
\left(\tau_{\text {eff }}^{\mathrm{ph}}\right)^{-1}=\frac{c}{l_{\mathrm{AMp}}}\left(1+\mathrm{Y}_{1}^{\mathrm{z}}+\mathrm{b} l_{\mathrm{AMp}} Y_{1}^{\mathrm{z}}\right)+\frac{c}{d_{\mathrm{AMp}}}\left(\mathrm{Y}_{1}^{\mathrm{y}}+\mathrm{a} d_{\mathrm{AMp}} Y_{1}^{\mathrm{y}}\right)
$$

Above relations show that we can define two independent effective cavity lifetimes for $z$ and $y$ directions,i.e.

$$
\begin{aligned}
& \left(\tau_{\mathrm{eff}}^{\mathrm{ph}, \mathrm{z}}\right)^{-1}=\frac{c}{l_{\mathrm{AMp}}}\left(1+{Y_{1}}_{1}^{\mathrm{z}}+\mathrm{b} l_{\mathrm{AMp}} Y_{1}^{\mathrm{z}}\right) \\
& \left(\tau_{\mathrm{eff}}^{\mathrm{ph}, \mathrm{y}}\right)^{-1}=\frac{c}{d_{\mathrm{AMp}}}\left(Y_{1}^{\mathrm{y}}+\mathrm{a} d_{\mathrm{AMp}} Y_{1}^{\mathrm{y}}\right)
\end{aligned}
$$

From Eq.(20) the overall effective cavity photon lifetime can be obtained

$$
\left(\tau_{\mathrm{eff}}^{\mathrm{ph}}\right)^{-1}=\left(\tau_{\mathrm{eff}}^{\mathrm{ph}, z}\right)^{-1}+\left(\tau_{\mathrm{eff}}^{\mathrm{ph}, y}\right)^{-1}
$$

Now we are able to plot the overall effective cavity photon lifetime of N2-lasers

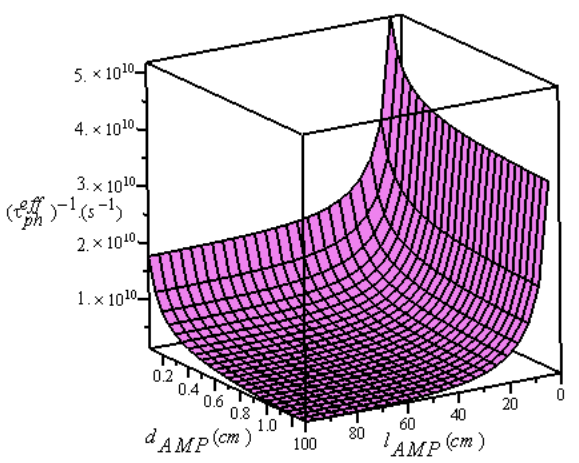

Fig4. Three dimensional profile of the inverse of effective cavity photon lifetime of N2-lasers

As we have an accurate relation for the overall effective cavity photon lifetime, with applying the corresponding relation and by using of experimental parameter deduced from [8], $b=0.042\left(\mathrm{~cm}^{-1}\right)$ and $\gamma_{l}^{\text {z.max }}=4.193$ (labeled data1), plus, $\gamma_{l}^{\text {z.max }}=3.897, b=0.06\left(\mathrm{~cm}^{-1}\right)[7]$ (labeled data2), we can draw the profile corresponding to use of first term appeared in the left side of Eq. (13).Using $a=0.82\left(\mathrm{~cm}^{-1}\right)$ and $\gamma_{1}^{\mathrm{y} \cdot \mathrm{max}}=0.08$ [8] (labeled data 1), plus $\gamma_{1}^{\text {y.max }}=0.058, a=0.78\left(\mathrm{~cm}^{-1}\right)$ [7] (labeled data 2), we can draw profilecorresponding to use of second term appeared in the left side of Eq. (13).
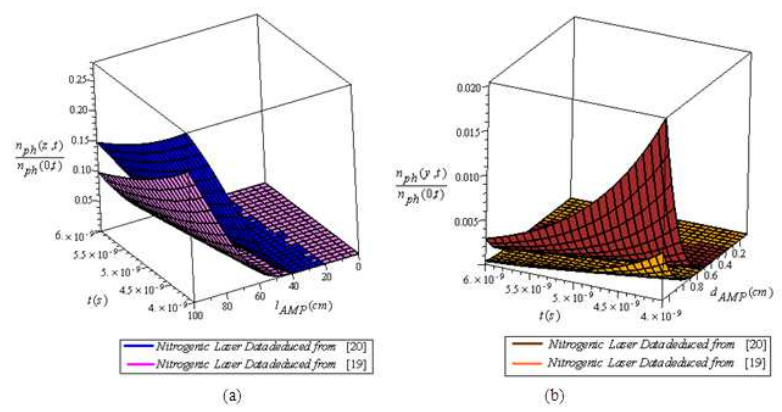

Fig 5.(a). three dimensional profile of photon density along $z$ direction ,or the length of the amplify medium $\left(z \cong l_{A M P}\right)$.(b): the three dimensional profile of photon density along $y$ direction, or the gap separation $\left(y \cong d_{A M P}\right)$.

\section{Conclusion and Discussion}

In this paper, based on experimental measurements for the effective photon lifetime in N2-laser, and the simulation study, we developed a model toexplain the dependency of this parameter on the geometrical dimensions given by $l_{A M P}$ and $d_{A M P}$, where they are referring to the AMP electrodes length and gap separation, respectively. It is shown that the model can be viewed according to one- and two dimensional approaches. We have shown that the effective photon lifetime obey the one-dimensionalequation of the type $\tau_{p h}^{\text {eff }}=\frac{\mathrm{l}_{\mathrm{AMP}}}{\mathrm{C}\left(1+\gamma_{1}-\mathrm{l}_{\mathrm{AMP}} \frac{\partial \gamma_{1}}{\partial \mathrm{l}_{\mathrm{AMP}}}\right)}=\frac{\mathrm{l}_{\mathrm{AMP}}}{\mathrm{C}\left(1+\gamma_{1}+\mathrm{b} \gamma_{1} \mathrm{l}_{\mathrm{AMP}}\right)}$, where $\mathrm{b}$ is some constant, This equation is indicating that for short $l_{A M P}$, the effective cavity photon lifetimedecreases. Our simulation studies using rateequations with different photon effective life time, give also a gainenhancement upon reducing the electrodes length, as given in Ref. [14]. In the two dimensional approach we have also shown that gain value in N2-lasers can be viewed as a two dimensional coefficientobeying photon density equations in the rate equations.

\section{References}

[1] E. Armandillo, A. Luches, V. Nassisi, M.R. Perrone, Appl. Opt. 24 (1985) 18.

[2] M. Csele, Fundamentals of light sources and lasers, John Wiley, 2004.

[3] F. Docchio, V. Magni, R. Ramponi, Rev. Sci. Instrum. 55 (1984) 477

[4] W.A. Fitzsimmons, L.W. Anderson, C.E. Riedhauser, Jan M. Vrtilek, IEEE, J. Quant. 
[5] L.M. Frantz, J.S. Nodvik, J. Appl. Phys. 34 (1963) 2346.

[6] M.C. Gower, C.B. Edwards, Opt. Commun. 40 (1982) 369.

[7] A. Hariri, S. Ghoreyshi, K. Rahimian, J. Appl. Phys. 101 (2007) 033132.

[8] A. Hariri, M. Jaberi, S. Ghoreyshi, Opt. Commun. 281 (2008) 3841.

[9] A. Hariri, S. Sarikhani. Optics Communications 284 (2011) 2153-2163

[10] H. K. Law, W O. Siew, K. K. Tham, and T. Y. Tou, An ultraviolet.

[11] A. V. Martinez and V. Aboites, Experimental optimization of a nitrogen laser, Instrumentation and development. 39 (1993), 396.
[12] A.D. Papadopoulos, A.A. Serafetinides, IEEE J. Quant. Electron. 26 (1990) 177.

[13] R. L. Roma and H. J. Ramos, Experimental investigation and characterization of a compact pulsed tea molecular nitrogen laser, Philippine Journal of Science. 2 (1996), 241254.

[14] S. Sarikhani, A. Hariri, Opt. Commun. 283 (2010) 118.

[15] W. T. Silfvast, Laser fundamentals, second edition, Cambridge University press, 2004.

[16] O. Svelto, Principles of laser, Springer Science and Business Media,Inc., 2009. 